\title{
Psychometric assessment of a cephalometric rubric in Dentistry undergraduate students
}

Gustavo González-Cuevas¹; José María Aguado²; Margarita Gómez²; Gleyvis Coro²

\section{ABSTRACT}

Educational practitioners in orthodontics have used cephalometric training as an instructional strategy for decades. Despite their widespread use, there is a lack of psychometrically sound assessment rubrics that researchers and practitioners can use to evaluate the quality of cephalometric training. To explore this issue, we developed and evaluated a new rubric tested in a sample of 175 dentistry undergraduate students at European University of Madrid. By applying reliability analysis, we found that our rubric demonstrated acceptable psychometric properties. We also observed that the internal structure of the rubric was unidimensional in our exploratory factorial analysis. We discuss implications for dentistry research and practice in relation with the use of modern psychometric tools.

\section{CONCLUSION}

All in all, the internal reliability and factorial validity demonstrated in this study support the conclusion that this cephalometric rubric may represent a useful tool for the objective and rigorous evaluation of cephalometric training in the Dentistry degree.

\section{INTRODUCTION}

With the development of the European Higher Education Area (Bologna, 1999), one of the most important challenges in the teaching methodologies was to establish a more rigorous evaluation. However, in the Dentistry degree, 20 years later, this process is still in progress. Indeed, rubrics are in need of a psychometrically sound assesment.
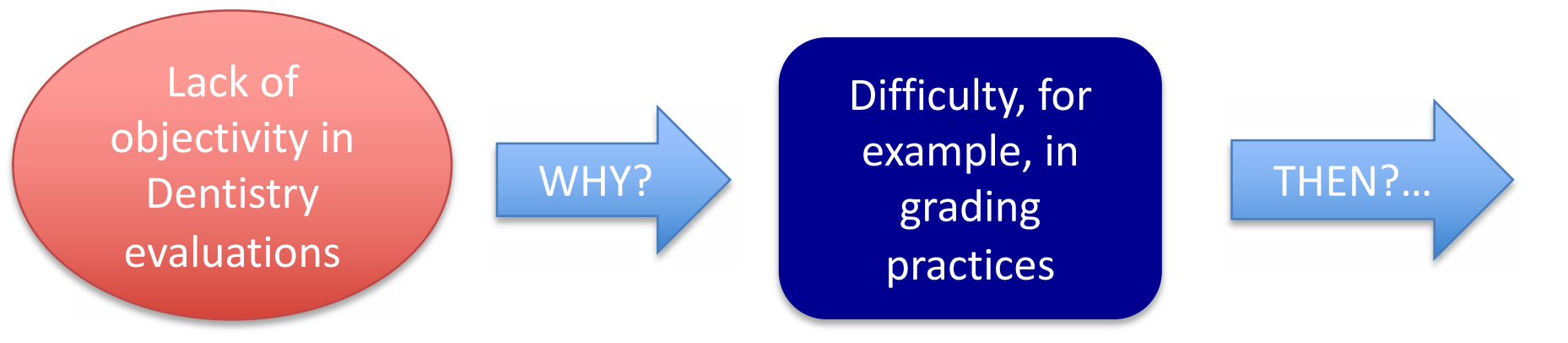

\section{RUBRICS \\ for practical evaluation}

\section{METHODOLOGY}

The faculty members of the Dentistry Department at European University of Madrid designed the overall guidelines for practical rubrics in the Dentistry degree. For the Orthodontics I course, the validation was carried out with external professors from other universities. After a pilot project with one group of students, a consensus of the final design for the cephalometric rubric was reached.

The rubric consists of 5 evaluation items:
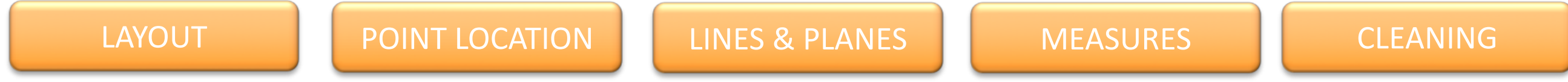

The psychometrics properties (reliability and validity) of the cephalometric rubric were tested in a sample of 175 Dentistry undergraduate students who enrolled in the Orthodontics I course at European University of Madrid and completed a cephalometric task.
RESULTS

\begin{tabular}{|l|c|c|c|}
\hline Item (47.1\% variance) & Mean & SD & $\begin{array}{c}\text { Factor } \\
\text { loadings }\end{array}$ \\
\hline Layout & 2.42 & 0.92 & 0.86 \\
\hline Point location & 3.11 & 0.94 & 0.60 \\
\hline Lines and planes & 2.86 & 1.07 & 0.53 \\
\hline Measures & 2.37 & 1.05 & 0.50 \\
\hline Cleaning & 2.82 & 0.94 & 0.40 \\
\hline
\end{tabular}

Table 1. Factor loadings for the cephalometric rubric: Principal Axis Factoring $(N=175)$

The average total score for the cephalometric task (Min: 0; Max: 10) was 6.57 (2.13) The average scores for the items (Min: 1 ; Max: 4$)$ were $3.11(0.92)$ for point location; 2.86 (1.07) for lines and planes; 2.82 (0.94) for cleaning; 2.42 (0.92) for layout; and 2.37 (1.05) for measures.

An exploratory factor analysis (eigenvalue $>1$ ) was conducted to evalute the dimensional structure of the cephalometric rubric. This analysis resulted in a one-factor solution that included all items and explained $47.1 \%$ of the total variance (Table 1 ).

For overall reliability of the total scale an aceptable Cronbach's alpha of .71 was observed. 